\title{
Periodic breathing in patients with stable obstructive sleep apnea on long-term continuous positive airway pressure treatment: a retrospective study using CPAP remote monitoring data
}

\author{
Kimimasa Saito $^{1} \cdot$ Yoko Takamatsu $^{1}$
}

Received: 6 May 2021 / Revised: 21 August 2021 / Accepted: 6 October 2021 / Published online: 14 October 2021

(c) The Author(s) 2021

\begin{abstract}
Purpose The purpose of this study was to investigate the rate of periodic breathing (PB) and factors associated with the emergence or persistence of PB in patients with obstructive sleep apnea (OSA) by continuous positive airway pressure (CPAP) remote monitoring data.

Methods This was a retrospective cohort study on 775 patients who had used the same model CPAP machine for at least 1 year as of September 1, 2020. The data were analyzed online using the dedicated analysis system. Using exporter software, average apnea/hypopnea index (AHI), average central apnea index (CAI), and average the rate of PB time (PB\%) were cited. Results Among 618 patients analyzed (age $61.7 \pm 12.2$ years, male 89\%, BMI 27.2 \pm 4.9 ), the average duration of CPAP use was $7.5 \pm 4.0$ years. The median PB\% in stable patients was low at $0.32 \%$, and only 149 patients (24\%) had a PB\% above $1 \%$. Multiple regression analysis of factors for the development of PB showed that the most important factor was atrial fibrillation (Af) with a coefficient of 0.693 (95\% CI; 0.536 to 0.851 ), followed by QRS duration with a coefficient of 0.445 (95\% CI; 0.304 to 0.586 ), followed by history of heart failure, male sex, comorbid hypertension, obesity, and age. The average PB\% for paroxysmal Af was significantly lower than that for persistent and permanent Af.

Conclusions The median PB\% in stable patients on CPAP treatment was low at $0.32 \%$, with only $24 \%$ of patients having $\mathrm{PB} \% \geq 1 \%$. Persistent Af and an increase in QRS duration were found to be important predictors of increased PB\%.

Clinical trial registration UMIN000042555 2021/01/01.
\end{abstract}

Keywords Cheyne-Stokes breathing · Periodic breathing · CPAP remote monitoring · Atrial fibrillation · QRS duration · Obstructive sleep apnea

\section{Introduction}

The current International Classification of Sleep Disorders (ICSD-3) classifies central sleep apnea (CSA) into 8 types based on the presence or absence of Cheyne-Stokes breathing (CSB) and other criteria [1]. However, the pathophysiological significance of CSA-CSB is still controversial.

This manuscript has not been published or presented elsewhere in part or in entirety and is not under consideration by another journal.

Kimimasa Saito

k1saito@ carrot.ocn.ne.jp

1 Saito Naika Kokyukika, Mie Sleep Clinic, Ise-shi 519-0502, Japan
Important predisposing factors of CSA-CSB include congestive heart failure (CHF), cerebrovascular disorder, and, probably, renal failure. In clinical practice, we often experience cases in which the frequency of CSA-CSB increases with exacerbation of left ventricular systolic heart failure and decreases or disappears with improvement of heart failure.

In other words, the occurrence and frequency of CSACSB may vary, depending on the disease conditions.

Since publication of the results of the SAVE-HF (Treatment of Predominant Central Sleep Apnea by Adaptive Servo-ventilation in Patients with Heart Failure) trial [2], a study demonstrating that adaptive servo-ventilation (ASV) does not alleviate nocturnal cardiovascular stress in patients with systolic heart failure and predominant CSA [3], the importance of CSA-CSB as a treatment target, particularly in patients with impaired left ventricular function, has been 
reduced. On the other hand, another study demonstrated that a reduction of pulmonary congestion as achieved by a decrease of left atrial pressure through successful MitraClip implantation is associated with the improvement of CSACSB [4].

In real-world clinical practice, we often experience that remote monitoring of periodic breathing (PB) may allow much earlier detection of new onset and exacerbation of pathological conditions, especially cardiovascular disease, in patients with OSA [5-7].

The role of CSA-CSB or PB as a prognostic marker rather than a therapeutic target in all patients per se is increasing.

The current study focused on PB in patients with stable obstructive sleep apnea (OSA) undergoing treatment with continuous positive airway pressure (CPAP) for long term.

With the final goal of predicting the onset or exacerbation of cardiovascular events by remote monitoring of PB\% in patients with OSA on CPAP, we retrospectively analyzed CPAP remote monitoring data to determine $\mathrm{PB} \%$ and identify factors associated with the emergence or persistence of $\mathrm{PB}$ in patients with stable OSA on CPAP.

\section{Methods}

\section{Participants}

We conducted a retrospective cohort study on 775 patients who had used the Dream Station Auto CPAP machine (Philips Respironics, Murrysville, PA) for at least 1 year at Mie Sleep Clinic as of September 1, 2020. All patients were diagnosed as having OSA, with an apnea-hypopnea index (AHI) $\geq 20$ by overnight PSG, or having sleep apnea syndrome (SAS), predominantly of obstructive type, with $\mathrm{AHI} \geq 40$ by out-of-center sleep testing (OCST), and have been continuously treated with CPAP.

Exclusion criteria were as follows: age $\geq 90$ years as of September 1, 2020; poorly adherent patients with a percentage of days of CPAP use of $<50 \%$ or an average CPAP use time of $<4 \mathrm{~h}$ between September 1 and 30, 2020; and new onset of heart failure, worsening of chronic heart failure, or new onset or relapse of atrial fibrillation (Af) or other cardiovascular diseases in the 3 months before or after September 1, 2020 (i.e., between June 1, 2020, and November $30,2020)$.

This study was approved by the ethics committee of the Medical Corporation MSC ( $\# 20,002)$. Written consent was obtained from all patients. This study is registered in the UMIN Clinical Trials Registry (UMIN000042555).

The primary endpoint was the calculation of standard data, such as the monthly median PB\%, and the analysis of factors associated with PB in patients with stable OSA on
CPAP. The secondary endpoint was subgroup analyses of factors that were strongly associated with PB.

\section{Clinical data collection}

The following baseline characteristic data were extracted from the Medical Corporation MSC Mie Sleep Clinic database, which contained data from October 1, 2007, to November 30, 2020, with the base point set at September 1, 2020 (Table 1):

- Age, sex, BMI

- Average number of years of CPAP use

- Smoking status (former smoker, current smoker, or nonsmoker)

- Frequency of alcohol consumption (daily, $\geq 3 /$ week, $1-2 /$ week, $<1 /$ week, rarely, or never)

- QRS width on ECG within 1 year

Table 1 Baseline characteristics

\begin{tabular}{ll}
\hline Variables & OSA (n=618) \\
\hline Male (\%) & $549(89)$ \\
Age, year & $61.7 \pm 12.2$ \\
BMI, kg/m ${ }^{2}$ & $27.2 \pm 4.9$ \\
Usage years of CPAP, year & $7.5 \pm 4.0$ \\
Smoking status, $\mathrm{n}(\%)$ & \\
$\quad$ Current smoker & $99(16)$ \\
$\quad$ Former smoker & $269(44)$ \\
$\quad$ Nonsmoker & $250(41)$ \\
Frequency of alcohol consumption, $\mathrm{n}(\%)$ & \\
$\quad \geq 3$ times/wk & $148(24)$ \\
$\quad$ 1-2 times/wk & $97(16)$ \\
$\quad<1$ times/wk & $58(9)$ \\
$\quad$ Rarely or nevever & $315(51)$ \\
History of congestive heart failure & $29(5)$ \\
History of ischemic heart disease & $45(7)$ \\
Prior stroke/TIA & $31(5)$ \\
Chronic kidney disease above stage3b & $28(5)$ \\
$\quad$ eGFR(Cr), mL/min/1.73m ${ }^{2}$ & $71.0 \pm 16.0$ \\
Artrial fibrillation & $60(10)$ \\
Hypertension & $300(49)$ \\
Type 2 diabetes & $112(18)$ \\
Dyslipidemia & $206(33)$ \\
Chronic obstructive pulmonary disease & $156(25)$ \\
Pacemaker implanted & $7(1)$ \\
QRS duration on ECG,ms & $89.7 \pm 13.8$ \\
Central nervous system agents & $45(7)$ \\
\hline & \\
\hline &
\end{tabular}

Data are presented as $\mathrm{n}(\%)$ or average $\pm \mathrm{SD}$

$B M I$, body mass index; $C P A P$, continuous positive airway pressure; $T I A$, transient ischemic attack; $E C G$, electrocardiogram 
Data on comorbidities and patients' medication status were obtained from the database or from the medical records of patients following physician referral. The diagnoses of heart failure, ischemic heart disease, and Af were based on diagnoses by cardiologists at neighboring flagship hospitals.

- Comorbidities and past medical history

History of congestive heart failure, ischemic heart disease, Af, pacemaker (including cardiac resynchronization therapy (CRT) device) implantation, prior stroke/ transient ischemic attack (TIA), hypertension, diabetes, dyslipidemia, stage $\geq 3 \mathrm{~b}$ CKD, and chronic obstructive pulmonary disease.

- Medications

Table 2 shows the further classification of Af type (paroxysmal, persistent, or permanent), QRS complex, and central nervous system (CNS) agents. We included four medication classes as CNS agents: opioids, benzodiazepines, sedatives, and non-benzodiazepine agonists.

\section{Polysomnography}

Full attended overnight polysomnography (PSG) was conducted in a laboratory setting, which was classified as type 1 sleep study by the American Academy of Sleep Medicine (AASM). PSG was performed using the Alice $® 4$ or Alice $®$ 6 system (Philips Respironics, Murrysville, PA). Sleep and respiratory event scoring was carried out according to the AASM manual. Our clinic is specialized in pulmonary

Table 2 Sleep variables detected by CPAP remote monitoring during 1 month $(n=618)$

\begin{tabular}{ll}
\hline Variables & \\
\hline CPAP usage time, min & $381.0 \pm 74.5$ \\
CPAP usage day, $\%$ & $95.5 \pm 9.4$ \\
AHI, no/hr & $3.1(0.2-36.6)$ \\
$\quad$ Log AHI & $0.48 \pm 0.31$ \\
CAI,no/hr & $0.12(0-15.6)$ \\
Log CAI & $-0.83 \pm 0.48$ \\
PB\%Device, $\%$ & $0.32(0-32.8)$ \\
75th percentile PB, $\%$ & 0.94 \\
$\quad$ Avbove $1 \%$ & $149(24.1)$ \\
Log PB\%Device & $-0.43 \pm 0.65$ \\
Large leak, $\%$ & $0.52(0-55.3)$ \\
$\quad$ Log Large leak & $-0.03 \pm 0.82$ \\
\hline
\end{tabular}

Data are presented as average $\pm \mathrm{SD}$, median (range),75th percentile or n $(\%)$

$C P A P$, continuous positive airway pressure; $A H I$, apnea/hypopnea index;

$C A I$, clear airway (central apnea) index; $P B$, periodic breathing medicine. All patients with a history of heart failure underwent diagnostic PSG in the stable compensatory phase of heart failure.

\section{Definitions of PB\% detected during diagnostic PSG and by the CPAP device}

The AASM recommends scoring a respiratory event as Cheyne-Stokes breathing if both of the following criteria are met:

a. There are episodes of at least three consecutive central apneas and/or central hypopneas separated by a crescendo and decrescendo change in breathing amplitude, with a cycle length of at least $40 \mathrm{~s}$ (typically 45 to $90 \mathrm{~s}$ ).

b. There are five or more central apneas and/or central hypopneas per hour associated with the crescendo/decrescendo breathing pattern recorded over a minimum of $2 \mathrm{~h}$ of monitoring.

According to the algorithm of the CPAP device, the device detects PB that meets only criterion a. Thus, in this study, we defined PB as follows:

PB\%PSG, defined as the percentage (\%) of the time exhibiting PB to total sleep time (TST) during diagnostic PSG

PB\%Device, defined as the percentage (\%) of the time exhibiting PB to the duration of the CPAP device use recorded in the CPAP data

Patients with $\mathrm{PB} \%$ Device $\geq 2 \%$ were divided into the emergence group that included those with PB $\%$ PSG $<1 \%$ and the persistence group that included those with $\mathrm{PB} \% \mathrm{PSG} \geq 1 \%$.

The clinical factors associated with the emergence or persistence of PB were investigated in these groups.

\section{CPAP titration}

At our clinic, CPAP treatment is initiated in the Auto CPAP mode on an outpatient basis (Supplemental Figure). When the treatment is initiated, the upper limit of pressure is set at a value estimated to not be excessive, according to the body weight of each patient. Within 1 week of treatment initiation, daily remote monitoring was initiated. The association between pressure variation and respiratory events, as well as leak patterns, is closely monitored, in addition to the AHI calculated using the CPAP device. The patient was re-examined at the outpatient clinic within 2-4 weeks after treatment initiation. If obstructive events persist $(\mathrm{AHI} \geq 10, \mathrm{~PB} \%$ Device $<2 \%$ ) after the set pressure upper limit was reached, we would raise the pressure limit further. 
When a patient exhibits PB and a large leak with pressure reaching the upper limit or increasing despite an $\mathrm{AHI} \geq 10$, $\mathrm{PB} \%$ Device $\geq 2 \%$, and a decreased incidence of obstructive events, the patient is regarded to have treatment-emergent central sleep apnea (TECSA). The pressure at the occurrence of $\mathrm{PB}$ was assumed to be the break point, and the upper limit of the pressure was adjusted to be lower than this point. This adjustment leads to resolution of the TECSA pattern within 3 months in most patients without comorbid heart or cerebrovascular disease. When the TECSA pattern persists for 3 months, patients are admitted for manual CPAP titration under PSG. Meanwhile, patients suspected of having comorbid heart failure or other heart or cerebrovascular diseases, and who provided consent, underwent measurement of brain natriuretic peptide (BNP), electrocardiography, and echocardiography, according to different criteria (AHI15 and CSR > 2\%). The patients were then admitted for manual adaptive servo-ventilation (ASV) titration under PSG within 3 to 6 months. Patients indicated for ASV are transitioned to ASV.

CHF patients with TECSA pattern sets the minimum pressure support of the ASV to $0 \mathrm{cmH} 2 \mathrm{O}$. This study included patients who had been continuously treated with CPAP for 1 year or longer, after undergoing the procedure described above at our clinic.

\section{Sleep data collection}

The CPAP device used was Dream Station Auto. The device periodically records the user's breathing signals and analyzes the data in near real time. It also records raw respiratory data at random. Analysis results are automatically uploaded to a central database. The data were analyzed online using the dedicated analysis system (EncoreAnywhere, ver. 2.44; Philips Respironics) and cited using the CareExporter software (version 1.11.1.0; Philips Respironics). The data cited were average apnea/hypopnea index (AHI), average central apnea (clear airway) index (CAI), PB\%Device, average percentage of large leak on days of CPAP use (LL\%), percentage of days of CPAP use (\%), and average CPAP use time (on days of use, $\mathrm{min}$ ). The monitoring data from the CPAP device was averaged over a 30-day period starting on September $1,2020$.

\section{Statistical analysis}

The Kolmogorov-Smirnov test was used to test the normal distribution of continuous variables. Data are presented as average $\pm \mathrm{SD}$, median (range), 75th percentile, or $n(\%)$. The data for AHI, CAI, PB\%Device, and LL\% showed a pointed distribution, expressed as median (full range), and transformed to 10-based logarithmic scale to obtain a normal distribution. The correlation among log AHI, log
CAI, and log PB\%Device was analyzed using the Pearson product-moment correlation coefficient. For the analysis of factors associated with $\mathrm{PB}$, multiple regression analysis was used to create a prediction formula for log PB\%Device. For explanatory variables, those that were considered to be clinically important based on previous studies were added to the model. In addition to age and sex, smoking history and obesity, which are cardiovascular risk factors, as well as factors previously identified as being associated with $\mathrm{PB}$, including use of CNS agents, such as alcohol and opioid $[8,9]$, history of congestive heart failure, history of stroke/ TIA, history of pacemaker implantation, Af, hypertension, concomitant stage $\geq 3$ b CKD [10], QRS duration on ECG [11-13], percent large leak calculated from CPAP device data [7, 14], and average CPAP use time [15], were considered to be related to $\mathrm{PB}$ onset and analyzed as binary variables, where $\mathrm{BMI} \geq 30, \mathrm{CKD}$ stage $\geq 3 \mathrm{~b}, \mathrm{QRS} \geq 110 \mathrm{~ms}$, and $L L \% \geq 15 \%$ were coded as 1 . The difference in average $\log$ PB\%Device between different Af types was analyzed using one-way analysis of variance (ANOVA), followed by multiple comparison using Tukey's post hoc test. The difference in QRS duration between the two groups was analyzed using Welch's $t$-test.

Probability values $\leq 0.05$ were considered significant. All statistical analyses were performed with EZR Ver.1.52 (Saitama Medical Center, Jichi Medical University, Saitama, Japan), which is a graphical user interface for the $\mathrm{R}$ version 4.02 (The R Foundation for Statistical Computing, Vienna, Austria). Specifically, it is a modified version of the $\mathrm{R}$ commander designed to add statistical functions frequently used in biostatistics.

\section{Results}

\section{Baseline characteristics}

A total of 775 patients were enrolled, of whom 3 were aged $\geq 90$ years, 149 were poorly adherent to CPAP treatment, and 5 had cardiovascular events within 3 months before or after analysis. Consequently, 618 patients were considered as having stable OSA and were included in the analysis (Fig. 1). Table 1 lists the baseline characteristics of the analyzable patients. The average duration of CPAP use by the 618 patients was $7.5 \pm 4.0$ years ( $1-13$ years). The average age was $61.7 \pm 12.2$ years, men accounted for $88.8 \%$ of all patients, and the average BMI was $27.2 \pm 4.9 \mathrm{~kg} / \mathrm{m}^{2}$. A history of congestive heart failure was seen in $5 \%$ of patients, while 5\% had prior stroke/TIA and 5\% had stage $\geq 3 \mathrm{~b}$ CKD. Of the $60(10 \%)$ patients with Af, 25 had paroxysmal, 10 had persistent, and 25 had permanent type of Af. The QRS cut-off of $110 \mathrm{~ms}$ was based on the upper limit of the normal range for the Japanese population. Of the 16 patients 
Fig. 1 Flow diagram of the study subject selection process. $C P A P$, continuous positive airway pressure

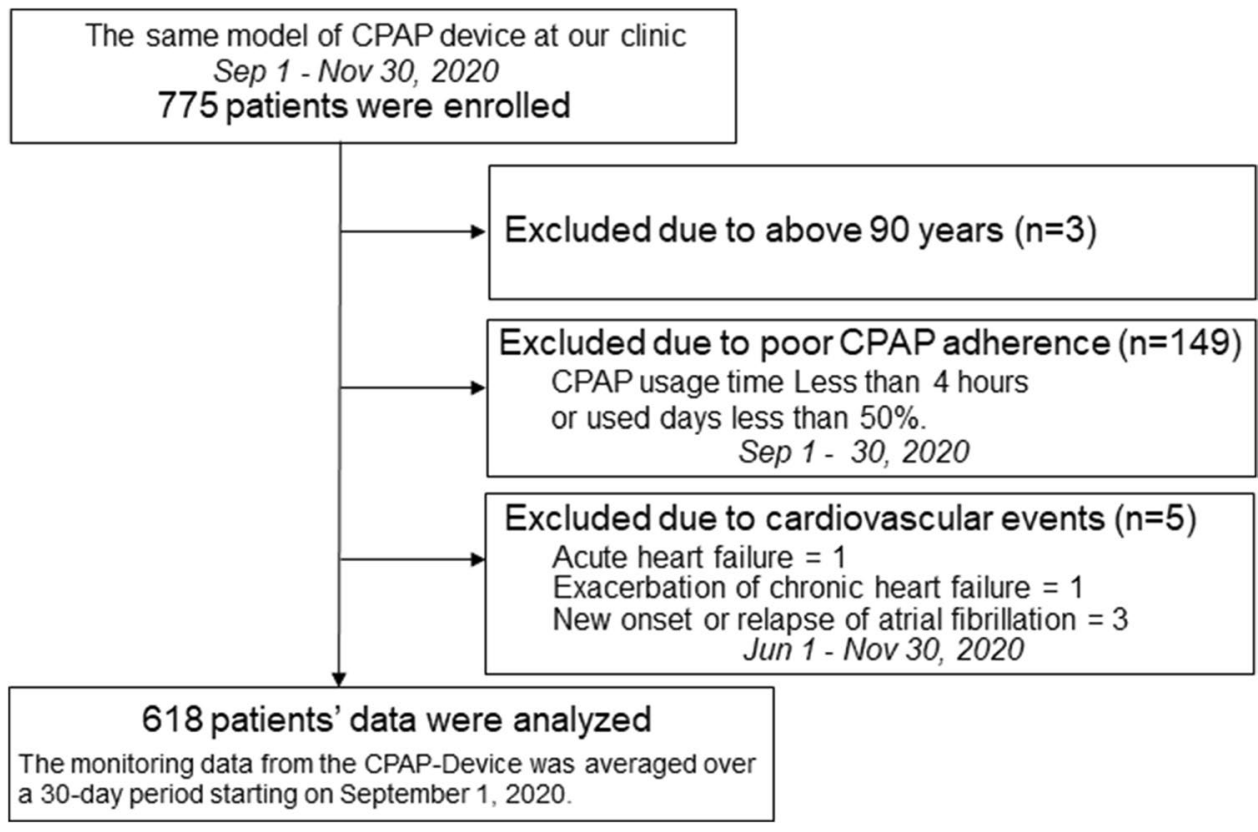

with a QRS duration $\geq 120 \mathrm{~ms}, 14$ had complete right bundle branch block (CRBBB), including 1 patient with Wolff-Parkinson-White syndrome. Among CNS agents, opioids were used only by 2 patients, both for pain relief from orthopedic diseases (Supplemental Table).

\section{Sleep variables detected by CPAP remote monitoring}

Sleep variables derived from the CPAP device are shown in Table 2. The average CPAP use time was 381.0 $\pm 74.4 \mathrm{~min}$, and the average percentage of days of CPAP use was $95.5 \pm 9.4 \%$, indicating excellent adherence to CPAP treatment. The median AHI of 3.1 times/h also indicates favorable control of SAS in almost all patients. The median PB\%Device in stable OSA patients on CPAP was low at $0.32 \%$ with a 75 th percentile of $0.94 \%$. The average log PB\%Device was $-0.43 \pm 0.64$, which corresponds to a PB\%Device of $0.37 \% \pm 2.360(0-32.8 \%)$. Thus, of the 618 patients, 149 (24\%) had $\mathrm{PB} \%$ of $\geq 1 \%$.

\section{Relationship between indexes of apnea event and $\log$ PB\%}

Figure $2 \mathrm{a}$ and $\mathrm{b}$ show the correlations between $\log \mathrm{AHI}$ and $\log$ PB\%Device and between $\log$ CAI and $\log$ PB\%Device, respectively. Patients with $\mathrm{AHI} \geq 5$ with poorly controlled OSA on CPAP tended to have higher PB\%Device values and showed a moderate correlation between AHI and PB\%Device with $r=0.562$ (95\% CI: 0.51-0.61). Of the 466 patients whose SAS was well controlled with AHI $<5,66$ (14.2\%) had PB\%Device $\geq 1 \%$, with the minimum being $1 \%$ and the maximum being $14.3 \%$. The correlation between log CAI and $\log$ PB\%Device was moderate with $r=0.42$, and only 2 patients had $\mathrm{CAI} \geq 5$.

\section{Multiple linear regression analysis for log PB\%Device $(n=607)$}

The results of linear multiple regression analysis of factors associated with PB (log PB\%Device) are shown in Table 3. Eleven patients had a PB\% of 0 , which was considered as missing data. The $p$ value of $F$-test was $<0.001$, indicating that the current model was effective. The most important factor was atrial fibrillation (Af) with a coefficient of 0.693 (95\% CI; 0.536 to 0.851 ), followed by QRS duration coefficient of 0.445 (95\% CI; 0.304 to 0.586 ), followed by history of heart failure, male, comorbid hypertension, obesity, and age. Contrastingly, CKD, prior stroke/TIA, and CNS agents were not strongly associated with CSB onset. Finally, the following regression equation was obtained.

Equation for multiple regression: $[\log \mathrm{PB} \%$ Device $=-$ $1.464+0.011 \times$ age $+0.213 \times$ male $(1)+0.136 \times \mathrm{BMI} \geq 30$ $(1)+0.693 \times$ Atrial fibrillation $(1)+0.227 \times$ History of $\mathrm{CHF}$ (1) $+0.143 \times$ Hypertension $(1)+0.445 \times \mathrm{QRS}$ duration $\geq 110$ (1)].

\section{Comparison of $\log$ PB\% and log AHI from the CPAP devices: paroxysmal Af group vs. persistent Af group vs. permanent Af group}

In the comparison of $\log \mathrm{PB} \%$ Device by type of Af, no significant difference was observed between permanent (average $\mathrm{PB} \%=6.81, n=25$ ) and persistent (average $\mathrm{PB} \%=2.37$, 


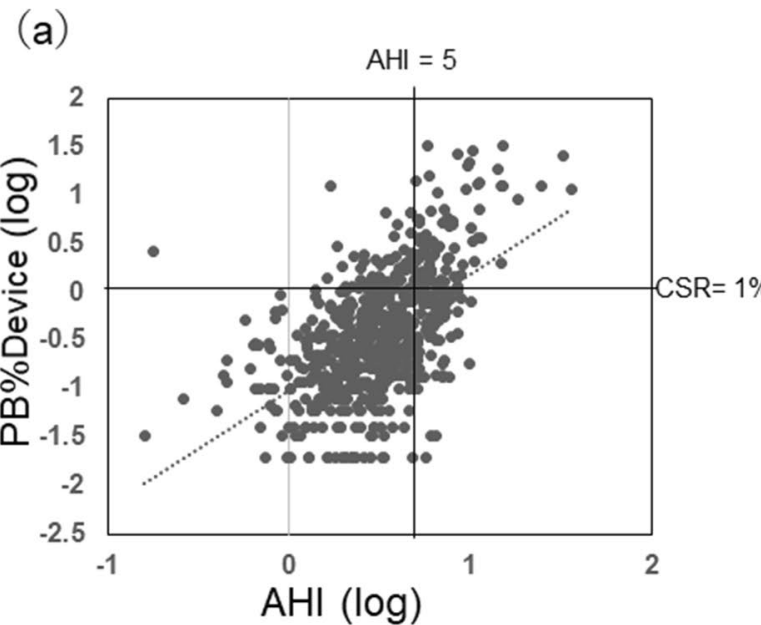

$r=0.562,95 \% \mathrm{Cl} 0.505-0.614, p<0.001$

Fig. 2 Relationship between indexes of apnea event and $\log$ PB\% from the CPAP devices. PB\%Device are plotted against their log $\mathrm{AHI}(\mathbf{a})$ and $\log \mathrm{CAI}(\mathbf{b})$. Note the significant correlation between $\log$

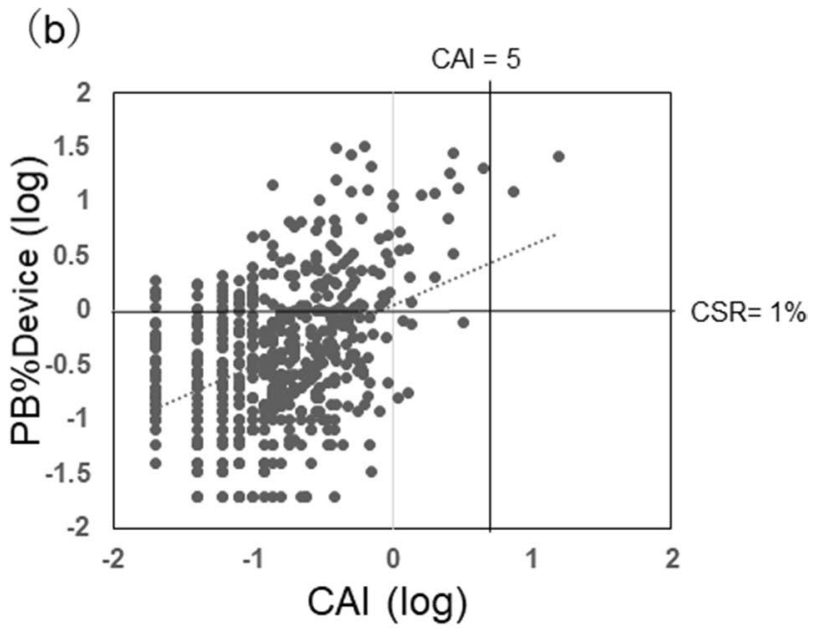

$r=0.418,95 \% \mathrm{Cl} 0.346-0.485, p<0.001$

PB\%Device and log AHI $(r=0.562, p<0.001), \log$ CAI $(r=0.418$, $p<0.001) . P B$, periodic breathing; $C P A P$, continuous positive airway pressure; $A H I$, apnea/hypopnea index; $C A I$, central/clear apnea index
Table 3 Multiple linear regression analysis for $\log$ PB\%Device $(n=607)$

\begin{tabular}{llllrl}
\hline Factor variables & Coefficient $(95 \% \mathrm{CI})$ & SE & t value & P value & vif \\
\hline Intercept) & $-1.464(-1.766$ to -1.161$)$ & 0.154 & -9.502 & $<\mathbf{0 . 0 0 1}$ & \\
Age & $\mathbf{0 . 0 1 1}(\mathbf{0 . 0 0 6}$ to $\mathbf{0 . 0 1 5})$ & 0.002 & 5.008 & $<\mathbf{0 . 0 0 1}$ & 1.345 \\
Male & $\mathbf{0 . 2 1 3}(\mathbf{0 . 0 5 7}$ to $\mathbf{0 . 3 6 8})$ & 0.079 & 2.680 & $\mathbf{0 . 0 0 8}$ & 1.231 \\
BMI $\geq 30$ & $\mathbf{0 . 1 3 6}(\mathbf{0 . 0 1 9}$ to $\mathbf{0 . 2 5 4})$ & 0.060 & 2.276 & $\mathbf{0 . 0 2 3}$ & 1.233 \\
Alcohol consumption & $0.010(-0.083$ to 0.103$)$ & 0.047 & 0.213 & 0.831 & 1.091 \\
Smoking status & $-0.046(-0.140$ to 0.048$)$ & 0.048 & -0.953 & 0.341 & 1.140 \\
Atrial fibrillation & $\mathbf{0 . 6 9 3}(\mathbf{0 . 5 3 6}$ to $\mathbf{0 . 8 5 1})$ & 0.08 & 8.658 & $<\mathbf{0 . 0 0 1}$ & 1.170 \\
History of CHF & $\mathbf{0 . 2 2 7}(\mathbf{0 . 0 3 1}$ to $\mathbf{0 . 4 2 3})$ & 0.100 & 2.274 & $\mathbf{0 . 0 2 3}$ & 1.460 \\
Hypertension & $\mathbf{0 . 1 4 3}(\mathbf{0 . 0 4 9}$ to $\mathbf{0 . 2 3 7})$ & 0.048 & 2.995 & $\mathbf{0 . 0 0 3}$ & 1.174 \\
Prior stroke/TIA & $0.029(-0.173$ to 0.232$)$ & 0.103 & 0.285 & 0.776 & 1.058 \\
CKD above stage3b & $-0.011(-0.223$ to 0.202$)$ & 0.108 & -0.097 & 0.923 & 1.057 \\
History of IHD & $-0.142(-0.327$ to 0.043$)$ & 0.094 & -1.503 & 0.133 & 1.249 \\
Pacemaker implanted & $-0.28(-0.721$ to 0.161$)$ & 0.225 & -1.246 & 0.213 & 1.178 \\
QRS duration $\geq 110$ & $\mathbf{0 . 4 4 5}(\mathbf{0 . 3 0 4}$ to $\mathbf{0 . 5 8 6})$ & 0.072 & 6.213 & $<\mathbf{0 . 0 0 1}$ & 1.073 \\
central nervous system agents & $-0.143(-0.310$ to 0.024$)$ & 0.085 & -1.680 & 0.093 & 1.019 \\
Large Leak $\geq 15 \%$ & $0.120(-0.074$ to 0.313$)$ & 0.098 & 1.216 & 0.224 & 1.050 \\
\hline
\end{tabular}

Regression coefficient values are presented with their 95\% confidence intervals (CI).

$* p$ values $\leq 0.05$ were considered significant. (statistical significance is indicated in bold)

Adjust $\mathrm{R}=0.536$ Adjusted $\mathrm{R}^{2}=0.287 \mathrm{~F} 15,591=17,27 p<0.001 \mathrm{~PB}$, periodic breathing; $S E$, standard error; $C I$, confidence interval; Vif, variance inflation factor; $B M I$, body mass index;

Alcohol consumption, 3 or more times per week; Smoking status, former or current smoker; $C H F$, congestive heart failureheart failure; TIA, transient ischemic attack; $C K D$, chronic kidney disease; $I H D$, ischemic heart disease

11 cases were missing PB\%Device at $0 \%$.

Equation for multiple regression: $[\log \quad \mathrm{PB} \%$ Device $=-1.464+0.011 \times$ age $+0.213 \times$ male $(1)+0.136 \times \mathrm{BMI} \geq 30(1)+0.693 \times$ Atrial fibrillation $(1)+0.227 \times$ History of CHF (1) $+0.143 \times$ Hypertension $(1)+0.445 \times \mathrm{QRS}$ duration $\geq 110$ (1)] 
$n=10$ ) types (Fig. 3a), while there were significant differences between paroxysmal (average PB $\%=0.69, n=25$ ) and persistent types $(p=0.032)$ and between paroxysmal and permanent types $(p<0.001)$. For log AHI, a significant difference between paroxysmal and permanent types $(p=0.002$; Fig. 3b) was observed.

\section{Comparison of $\log$ PB\% and $\log$ AHI from the CPAP devices: $120 \mathrm{~ms}>$ QRS duration $\geq 110 \mathrm{~ms}$ group vs. $Q R S$ duration $\geq 120$ ms group}

In comparisons between subgroups based on abnormal QRS duration on ECG, patients with QRS duration $\geq 120 \mathrm{~ms}$ group showed significantly higher $\log \mathrm{PB} \%$ Device values (average PB $\%: 3.16 \%, n=16$ ) than those with $120 \mathrm{~ms}>$ QRS duration $\geq 110 \mathrm{~ms}$ group (average $\mathrm{PB} \%: 0.82 \%, n=54$; $p=0.002$; Fig. $4 \mathrm{a}$ ). Similarly, $\log$ AHI values were also significantly higher in those with QRS duration $\geq 120 \mathrm{~ms}$ group (average AHI: 6.64, $n=16$ ) than those with $120>$ QRS duration $\geq 110$ ms group (average AHI: $3.59, n=54 ; p=0.003$; Fig. $4 b)$.

\section{Investigation of factors for CSB onset in the persistence group and the emergence group}

In 61 of 79 patients with $\mathrm{PB} \%$ Device $\geq 2 \%$, we re-examined the data from diagnostic PSG, calculated PB\%PSG,

(a)

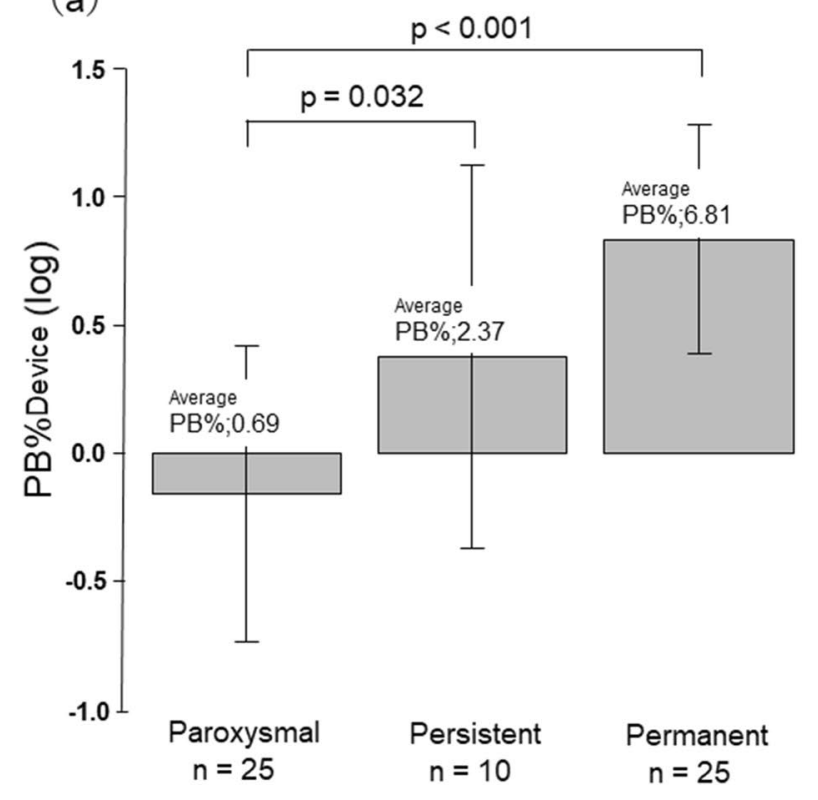

Fig. 3 Comparison of $\log \mathrm{PB} \%$ and $\log \mathrm{AHI}$ from the CPAP devices: paroxysmal Af group vs. persistent Af group vs. permanent Af group. Average PB\%Device, 0.69 vs. 2.37 vs. 6.81, respectively. Log PB\%Device was significantly higher in the persistent Af group and permanent Af group than in the paroxysmal Af group $(p=0.032$, and compared PB\%PSG with PB\%Device. Table 4 shows clinical factors of the persistence group and the emergence group.

In the persistence group, $\mathrm{PB} \% \mathrm{PSG}$ was $1 \%$ or higher at baseline in 37 of 61 patients $(60.7 \%)$. Of these 37 patients, 33 (89.2\%) showed at least one of the following findings: past history of CHF, stroke, and atrial fibrillation; diastolic dysfunction detected by echocardiography between 2019 and 2020; and increased QRS duration detected by electrocardiography between 2019 and 2020 (CHF in 13 patients, cerebral infarction in 5 patients, atrial fibrillation in 19 patients, diastolic dysfunction in 8 patients, and increased QRS duration in 13 patients). In the remaining four patients, elevated PAP device pressure was not associated with the occurrence of PB, and the frequency of PB indicated that TECSA was unlikely. Two of these patients were public transportation drivers, and their PB\% increased consistently on their days off; they had a habit of drinking a relatively large amount of alcohol on days off.

In the emergence group, PB\%PSG at the time of diagnosis was $<1 \%$ in 24 patients $(39.3 \%)$.

Of these 24 patients, $20(83.3 \%)$ showed at least one of the following findings during the period from diagnostic PSG to May 31, 2020: new onset or relapse of heart failure, cerebral infarction, or atrial fibrillation, diastolic dysfunction detected by echocardiography between 2019 and 2020, and increased QRS duration detected by electrocardiography

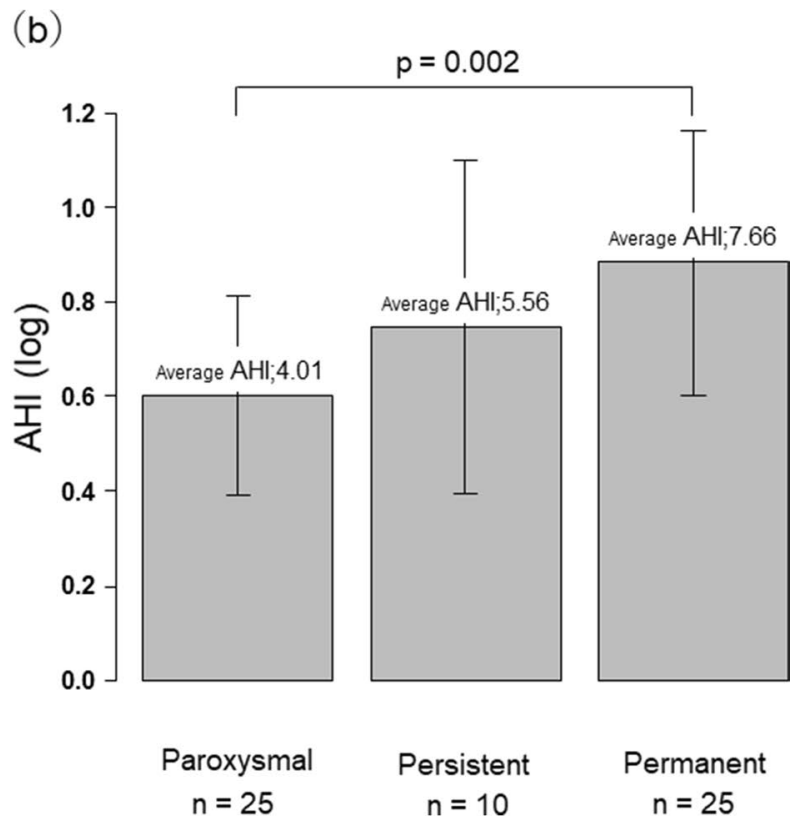

$p<0.001$ ). Average AHI, 4.01 vs. 5.56 vs. 7.66, respectively. Log AHI was significantly higher in the persistent Af group than in the paroxysmal Af group $(p=0.002)$. ${ }^{*} p \leq 0.05$, one-way ANOVA followed by Tukey's post hoc test. $P B$, periodic breathing; $A H I$, apnea/ hypopnea index 


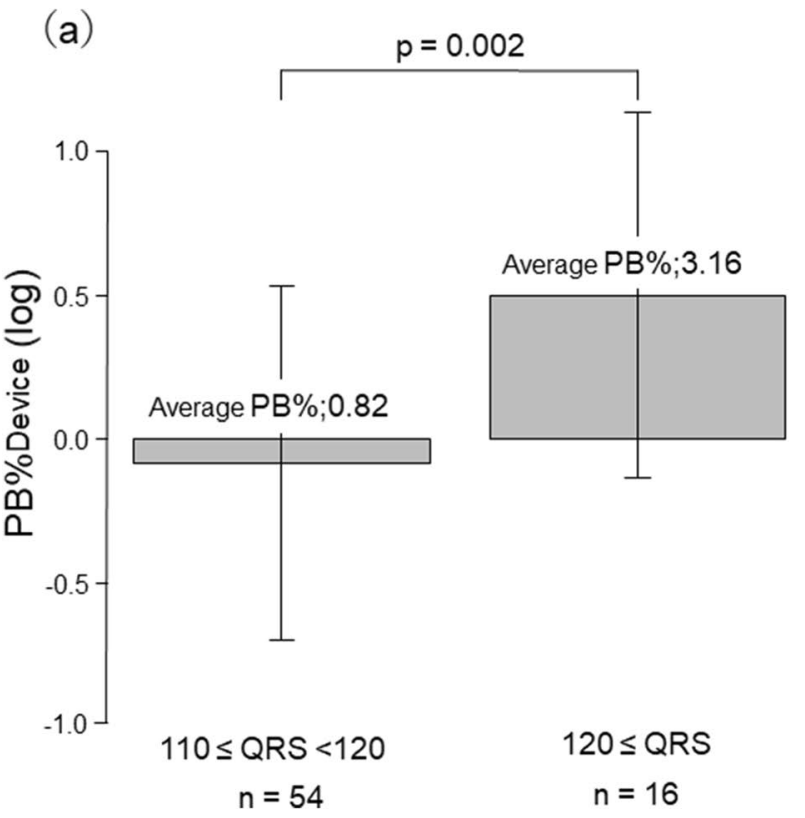

Fig. 4 Comparison of $\log$ PB\% and log AHI from the CPAP devices: $110 \leq$ QRS duration $<120$ group vs. $120 \leq$ QRS duration group. Average PB\%Device, 0.82 vs. 3.16, respectively. Log PB\%Device was significantly higher in the $120 \leq \mathrm{QRS}$ duration group than in the $110 \leq \mathrm{QRS}$ duration $<120$ group $(p=0.002)$. Average AHI,

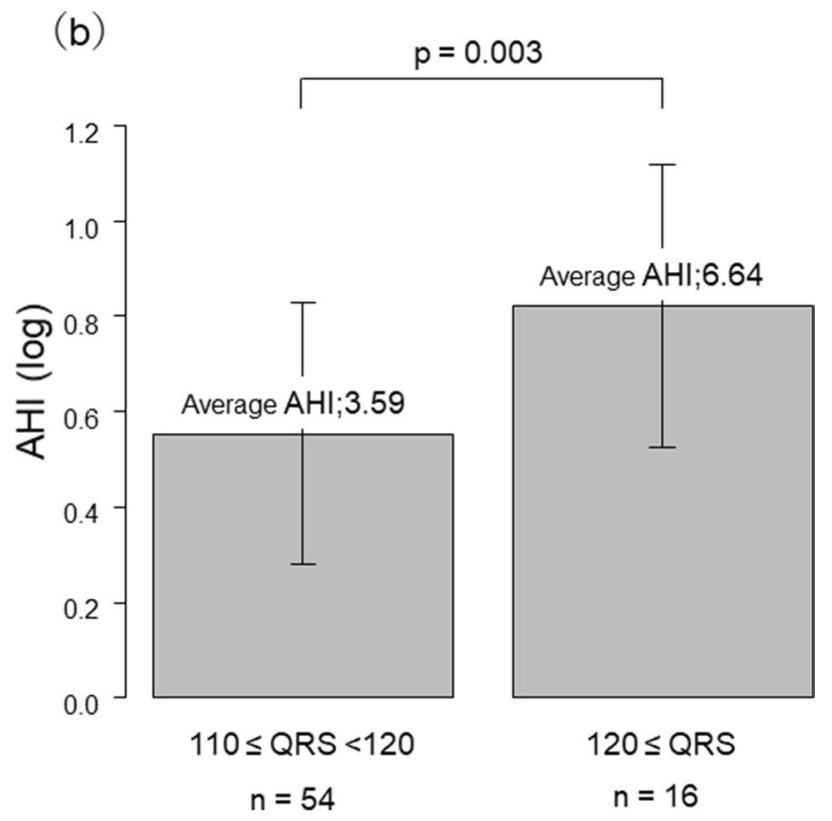

3.59 vs. 6.64 , respectively. Log AHI was significantly higher in the $120 \leq$ QRS duration group than in the $110 \leq$ QRS duration $<120$ group $(p=0.003)$. ${ }^{*} p \leq 0.05$, by Welch two-sample $t$-test. $P B$, periodic breathing; $A H I$, apnea/hypopnea index
Table 4 Clinical data of patients with $\mathrm{PB} \%$ Device $\geqq 2 \%(n=61)$

\begin{tabular}{lll}
\hline & Persistence & Emergence \\
\hline Variables & PB\%PSG $\geqq 1 \%(\mathrm{n}=37)$ & PB\%PSG < $\%(\mathrm{n}=24)$ \\
PB\%PSG & $8.1 \pm 8.0$ & $0.1 \pm 0.2$ \\
PB\%Device & $8.9 \pm 8.3$ & $4.3 \pm 3.1$ \\
Past History of Congestive heart failure & $13(35.1)$ & $5(20.8)$ \\
Exacerbation or new on set Congestive heart failure & & $\mathbf{6}(\mathbf{2 5 . 0})$ \\
Prior Stroke/TIA & $5(13.5)$ & $1(4.2)$ \\
New on set Stroke/TIA & & $\mathbf{1 ( 4 . 2 )}$ \\
Atrial fibrillation (comorbidity or relapse) & $19(51.4)$ & $\mathbf{7 ( 2 9 . 2 )}$ \\
QRS $>110$ ms & $13(35.1)$ & $\mathbf{1 0}(\mathbf{4 1 . 7})$ \\
Echocardiogram findings & & \\
Average E/e' & $14.0 \pm 4.1$ & $12.8 \pm 3.9$ \\
Average E/e' $>14$ & $8(21.6)$ & $\mathbf{4}(\mathbf{1 6 . 7})$ \\
Ejection fraction & $62.7 \pm 6.9$ & $64.3 \pm 5.7$ \\
Ejection fraction $\leqq 0.45$ & 0 & 0 \\
Left atrial dimension & $46.1 \pm 11.7$ & $40.3 \pm 7.4$ \\
Central nervous system agents & $2(5.4)$ & 0 \\
Alcohol consumption $\geq 3$ times/week & $10(27.0)$ & $9(37.5)$ \\
\hline
\end{tabular}

Data are presented as $\mathrm{n}(\%)$ or mean $\pm \mathrm{SD}$

$P B$, periodic breathing; $P S G$, polysomnography; TIA, transient ischemic attack

Echocardiogram Values missing for 14 patients with Persistence cases of PB

Echocardiogram Values missing for 12 patients with Emergence cases of PB 
between 2019 and 2020. (New onset or relapse of heart failure in 6 patients, new onset of cerebral infarction in 1 patient, comorbid atrial fibrillation in 7 patients, diastolic dysfunction in 4 patients, and increased QRS duration in 10 patients). In the remaining four patients, elevated PAP device pressure was not associated with the occurrence of PB. Their general condition was favorable, and their lifestyles did not change. The frequency of PB was approximately $2 \%$ in 3 of these 4 patients.

\section{Discussion}

We analyzed CPAP remote monitoring data to identify factors associated with the $\mathrm{PB}$ in patients with stable OSA on CPAP. No report describes PB\% in stable OSA patients on long-term CPAP treatment. This is the first report to derive the reference value and prediction formula for $\mathrm{PB} \%$.

In this cohort of stable patients with OSA without new cardiovascular events over 6 months, the median PB\% was low. Nevertheless we believe that PB\% has pathophysiological significance.

The correlation between $\log$ PB\%Device and $\log$ AHI was $r=0.56$. Even patients with $\mathrm{AHI}<5$, which indicated good control of OSA by CPAP, also had PB pattern. In actual clinical practice, patients are typically evaluated primarily by the AHI. Since in some cases the rise of PB\% is not reflected in the AHI, we believe that at the time of the SAS patient examination, PB\% must also be taken into consideration.

The prevalence of SAS in patients with chronic heart failure is reportedly $9-50 \%[16,17]$. Suggested predisposing factors for CSA-CSB include male sex, age $\geq 60$ years, the presence of Af, and daytime hypocapnemia [18]. In the current study involving patients with stable OSA, the most strongly predictive factors for increased $\mathrm{PB} \%$ were the presence of Af and QRS duration (especially CRBBB), followed by a history of congestive heart failure, male, comorbidity of hypertension, obesity, and age, similar to the predisposing factors of CSB in the general population reported by Donovan et al. [19].

The persistent and permanent types of Af were associated with significantly higher values of $\mathrm{PB} \%$ and $\mathrm{AHI}$ compared to the paroxysmal type. Similarly, an analysis by QRS duration also showed significantly higher values of PB\% and AHI in patients with QRS duration $\geq 120 \mathrm{~ms}$. In both comparisons, the difference was markedly higher for PB\%Device than AHI.

This study revealed that the PB patterns exhibited by the CPAP device were consistent with previously reported patterns of CSA-CSB observed under PSG.

In comparing PB\%PSG and PB\%Device, comorbidity of Af, history of CHF, and previous stroke contributed to the persistence of $\mathrm{PB} \%$. Recurrent or new onset of CHF, stroke or Af, and diastolic dysfunction and QRS width between the time of PAP implementation and May 31, 2020, were suggested to have an impact on the secular increase in PB\%.

These findings suggest that the PB detected by the device may be a useful clinical indicator.

An important pathophysiological factor for the onset of CSB is the response of various receptors involved in respiratory regulation. In patients with cardiac failure, increased left atrial pressure, pulmonary congestion, delayed circulation time, and increased sympathetic activity have been associated with CSB [20].

The association between CSB and Af has also been described [21]; Af is considered to cause CSA through a mechanism similar to congestive heart failure. Increasing pulmonary vascular resistance caused by the increased left atrial pressure stimulates the pulmonary vagal receptors, causing hyperventilation and hypopnea [22, 23].

Patients with Af, even with normal systolic left ventricular function, have been reported to have a higher prevalence of CSB [24], suggesting that CSB is more attributable to left ventricular end diastolic pressure or pulmonary congestion than to left ventricular systolic function itself.

Some authors have suggested that the presence of CSACSB predicts future onset of Af [25]. They state that the presence of CSA-CSB may contribute to increased sympathetic activity and autonomic imbalance, which are known predisposing factors of Af. We speculate that the presence of CSB itself indicates the presence of left atrial overload and pulmonary congestion. The left atrial overload may gradually increase over time, eventually leading to overt Af, and these are not sudden changes [26]. Therefore, we believe that time-course monitoring of PB\%Device is effective in predicting the onset of Af.

Regarding the association between CSB and QRS duration, we speculate the involvement of delayed circulation time and resulting delayed transmission of information to central chemical receptors. In patients with QRS duration $\geq 120 \mathrm{~ms}$, CRBBB was the most frequent cause of CSB onset. Decreased right ventricular function and right ventricular enlargement have been associated with the onset of CSB [27]. Delayed circulation time due to delayed or mismatched right ventricular wall motion may also contribute to CSB. Reduced CSB in patients undergoing CRT has also been described [28]. Among the 7 patients with a history of pacemaker implantation in the current study, some had CRT device implanted with reduced left ventricular ejection fraction, and CSA-CSB was hardly observed in these patients. Joseph et al. have concluded that prolonged QRS duration in patients with heart failure and preserved left ventricular contractility is associated with increased risk of adverse clinical outcomes, regardless of the type of conduction abnormalities responsible for QRS prolongation [29]. 
The current study is limited in that it was conducted retrospectively and at a single institution. It needs to be validated in a prospective multicenter study.

Second, no titration under PSG was performed before the initiation of CPAP treatment. We might have been unable to completely differentiate between TECSA and non-pathological CSA-CSB observed at sleep onset or in the rapid eye movement (REM) stage in elderly patients and others. Although there are various reports on the incidence of TECSA during the early phase of positive airway pressure (PAP) treatment, TECSA is self-limiting and resolves within 3 months in $90 \%$ or more of patients [30]. In a prospective study of patients with normal BNP levels, Westhoff et al. reported that the incidence of TECSA was as low as $0.56 \%$ [31]. We also consider that patients without heart disease can be treated to some extent, if remote monitoring is started soon after initiation of CPAP treatment to carefully observe them for the timing of pressure increase and PB onset, as well as the amount of leakage.

Third, because the data produced by the algorithm of the CPAP device were not fully validated, we were unable to detect central sleep hypopnea. However, if longitudinal monitoring of PB as a marker leads to early detection of heart disease, the algorithm may have great clinical benefit. In the future, the accuracy of the algorithm may need to be further improved through collaboration between the fields of medicine and engineering.

In conclusion, the median $\mathrm{PB} \%$ in stable OSA patients was low with only $24 \%$ of patients having PB $\% \geq 1 \%$. Furthermore, perpetuating factors of Af and an increase in QRS duration were identified as important predictors of increased PB $\%$.

Conversely, increased PB\%Device may predict the progression of pulmonary congestion that signals the onset and relapse of Af and heart failure.

Supplementary Information The online version contains supplementary material available at https://doi.org/10.1007/s11325-021-02510-0.

Author contribution Kimimasa Saito designed the study and wrote the initial draft of the manuscript. Yoko Takamatsu contributed to data collection, analysis, and interpretation of data and critically reviewed the manuscript. All authors approved the final version of the manuscript and agree to be accountable for all aspect of the work in ensuring that questions related to the accuracy or integrity of any part of the work are appropriately investigated and resolved.

Data availability The datasets used and/or analyzed during the study are available from the corresponding author on direct request.

Code availability Not applicable.

\section{Declarations}

Ethics approval This study was approved by the Institutional Review Board (IRB) of Medical Corporation MSC (\#20002, UMIN00000042555) and was carried out in accordance with the Declaration of Helsinki.

Consent to participate Written informed consent was obtained from participants.

Consent for publication All authors agree with the publication of our manuscript. Our manuscript is not under consideration for publication in any other journal. If accepted, the authors agree that the paper will not be published elsewhere in the same or a similar form, in English, or in any other language, without written consent of the copyright holder.

Competing interests The authors declare no competing interests.

Open Access This article is licensed under a Creative Commons Attribution 4.0 International License, which permits use, sharing, adaptation, distribution and reproduction in any medium or format, as long as you give appropriate credit to the original author(s) and the source, provide a link to the Creative Commons licence, and indicate if changes were made. The images or other third party material in this article are included in the article's Creative Commons licence, unless indicated otherwise in a credit line to the material. If material is not included in the article's Creative Commons licence and your intended use is not permitted by statutory regulation or exceeds the permitted use, you will need to obtain permission directly from the copyright holder. To view a copy of this licence, visit http://creativecommons.org/licenses/by/4.0/.

\section{References}

1. Darien IL (2014) International classification of sleep disorders, 3rd edition (ICSD-3) American Academy of Sleep Medicine

2. Cowie MR, Woehrle H, Wegscheider K, Angermann C, d'Ortho MP, Erdmann E, Levy P, Simonds AK, Somers VK, Zannad F, Teschler H (2015) Adaptive servo-ventilation for central sleep apnea in systolic heart failure. N Engl J Med 373:1095-1105

3. Gorbachevski M, Spiesshoefer J, Arzt M, Oldenburg O, Becker S, Tuleta I, Emdin M, Passino C, Sciarrone P, Boentert M, Giannoni A (2020) Adaptive servo-ventilation therapy does not favourably alter sympatho-vagal balance in sleeping patients with systolic heart failure and central apnoeas: Preliminary data. Int J Cardiol 315:59-66

4. Spiesshoefer J, Spieker M, Klose S, Keymel S, Boentert M, Krüger S, Horn P, Kelm M, Westenfeld RP (2019) Reduction of sleep-disordered breathing following effective percutaneous mitral valve repair with the MitraClip system. Sleep Breath 23:815-824

5. Light M, Orr JE, Malhotra A, Owens RL (2018) Continuous positive airway pressure device detects atrial fibrillation induced central sleep apnoea. Lancet 392(10142): 160

6. Schmickl CN, Heckman E, Owens RL, Thomas RJ (2019) The respiratory signature: a novel concept to leverage continuous positive airway pressure therapy as an early warning system for exacerbations of common diseases such as heart failure. J Clin Sleep Med 15(6):923-927

7. Prigent A, Serandour AL, Luraine R, Poineuf JS, Bosseau C, Pépin JL (2020) Interrelated atrial fibrillation and leaks triggering and maintaining central sleep apnoea and periodic breathing in a CPAP-treated patient. Respirol Case Rep 8(8):e00666 
8. Van Ryswyk E, Anderson CS, Antic NA (2016) Opioids and sleep-disordered breathing. Chest 150(4):934-944

9. Chakravorty S, Chaudhary NS, Brower KJ (2016) Alcohol dependence and its relationship with insomnia and other sleep disorders. Alcohol Clin Exp Res 40(11):2271-2282

10. Arzt M, Oldenburg O, Graml A, Erdmann E, Teschler H, Wegscheider K, Woehrle SA, H, (2017) Phenotyping of sleepdisordered breathing in patients with chronic heart failure with reduced ejection fraction-the SchlaHF registry. J Am Heart Assoc 6(12):e005899. https://doi.org/10.1161/JAHA.116.005899

11. Tinoco A, Mortara DW, Hu X, Sandoval CP, Pelter MM (2019) ECG derived Cheyne-Stokes respiration and periodic breathing are associated with cardiorespiratory arrest in intensive care unit patients. Heart Lung 48(2):114-120

12. Khalil MM, Rifaie OA (1998) Electrocardiographic changes in obstructive sleep apnoea syndrome. Respir Med 92(1):25-27

13. Kwon Y, Picel K, Adabag S, Vo T (2016) Sleep-disordered breathing and daytime cardiac conduction abnormalities on 12-lead electrocardiogram in community-dwelling older men. Sleep Breath 20(4):1161-1168

14. Montesi SB, Bakker JP, Macdonald M et al (2013) Air leak during CPAP titration as a risk factor for central apnea. J Clin Sleep Med 9(11):1187-1191

15. Javaheri S, McKane SW, Cameron N, Germany RE, Malhotra A (2019) In patients with heart failure the burden of central sleep apnea increases in the late sleep hours. Sleep 42(1):zsy195. https://doi.org/10.1093/sleep/zsy195

16. Ward NR, Roldao V, Cowie MR et al (2013) The effect of respiratory scoring on the diagnosis and classification of sleep disordered breathing in chronic heart failure. Sleep 36(9):1341-1348

17. Bitter T, Westerheide N, Hossain SM, Prinz C, Horstkotte D, Oldenburg O (2012) Symptoms of sleep apnoea in chronic heart failure-results from a prospective cohort study in 1,500 patients. Sleep Breath 16(3):781-791

18. Sin DD, Fitzgerald F, Parker JD, Newton G, Floras JS, Bradley TD (1999) Risk factors for central and obstructive sleep apnea in 450 men and women with congestive heart failure. Am J Respir Crit Care Med 160(4):1101-1106

19. Donovan LM, Kapur VK (2016) Prevalence and characteristics of central compared to obstructive sleep apnea: analyses from the sleep heart health study cohort. Sleep 239(7):1353-1359

20. Terziyski K, Draganova A (2018) Central sleep apnea with Cheyne-Stokes breathing in heart failure - from research to clinical practice and beyond. Adv Exp Med Biol 1067:327-351
21. Leung RS, Huber MA, Rogge T, Maimon N, Chiu KL, Bradley TD (2005) Association between atrial fibrillation and central sleep apnea. Sleep 28(12):1543-1546

22. Giannoni A, Raglianti V, Taddei C (2019) Cheyne-Stokes respiration related oscillations in cardiopulmonary hemodynamics in patients with heart failure. Int J Cardiol 289:76-82

23. Lorenzi-Filho G, Azevedo ER, Parker JD, Bradley TD (2002) Relationship of carbon dioxide tension in arterial blood to pulmonary wedge pressure in heart failure. Eur Respir J 19(1):37-40

24. Bitter T, Langer C, Vogt J, Lange M, Horstkotte D, Oldenburg O (2009) Sleep-disordered breathing in patients with atrial fibrillation and normal systolic left ventricular function. Dtsch Arztebl Int 106(10):164-170

25. May AM, Blackwell T, Stone PH (2016) Central sleep-disordered breathing predicts incident atrial fibrillation in older men. Am J Respir Crit Care Med 193(7):783-791

26. Bitter T, Fox H, Schmalgemeier $\mathrm{H}$ et al (2016) Acute improvement of pulmonary hemodynamics does not alleviate Cheyne-Stokes respiration in chronic heart failure-a randomized, controlled, double-blind, crossover trial. Sleep Breath 20(2):795-804

27. Christ M, Grimm W, Rostig S et al (2003) Association of right ventricular dysfunction and Cheyne-Stokes respiration in patients with chronic heart failure. J Sleep Res 12(2):161-167

28. Sinha AM, Skobel EC, Breithardt OA (2004) Cardiac resynchronization therapy improves central sleep apnea and Cheyne-Stokes respiration in patients with chronic heart failure. J Am Coll Cardiol 44(1):68-71

29. Joseph J, Claggett BC, Anand IS et al (2016) QRS duration is a predictor of adverse outcomes in heart failure with preserved ejection fraction. JACC Heart Fail 4(6):477-486

30 BaHammam AS (2017) Treatment-emergent central sleep apnea (complex sleep apnea). Sleep Vigilance 1:53-56. https://doi.org/ 10.1007/s41782-017-0015-8

31. Westhoff M, Arzt M, Litterst P (2012) Prevalence and treatment of central sleep apnoea emerging after initiation of continuous positive airway pressure in patients with obstructive sleep apnoea without evidence of heart failure. Sleep Breath 16(1):71-78. https://doi.org/10.1007/s11325-011-0486-0

Publisher's note Springer Nature remains neutral with regard to jurisdictional claims in published maps and institutional affiliations. 